\title{
Heavy flavour in high-energy nuclear collisions: overview of transport calculations
}

\author{
Andrea Beraudo*i \\ INFN - Sezione di Torino \\ Via pietro Giuria 1 - 10125 Torino - Italy \\ E-mail: beraudo@to.infn.it
}

\begin{abstract}
Transport calculations are the tool to study medium modifications of heavy-flavour particle distributions in high-energy nuclear collisions. We give a brief overview on their state-of-the art as well as on the questions remaining open, from the evaluation of the transport coefficients to the effects of in-medium hadronization, from the rescattering in the hadronic phase to the possible presence of hot-medium effects also in small systems, like proton-nucleus collisions.
\end{abstract}

The European Physical Society Conference on High Energy Physics

5-12 July, 2017

Venice

\footnotetext{
* Speaker.

${ }^{\dagger}$ Thanks to my collaborators A. De Pace, M. Monteno, M. Nardi and F. Prino
} 


\section{Introduction}

The description of heavy-flavour observables in relativistic heavy-ion collisions requires to develop an involved multi-step setup. One has to simulate the initial $Q \bar{Q}$ production in the hard process and for this automated QCD event generators - to validate against proton-proton data - are available, possibly supplemented with initial-state effects, like nuclear Parton Distribution Functions. One has then to rely on a description of the background medium, in particular its temperature and four-velocity, provided by hydrodynamic calculations tuned to reproduce soft-hadron data. One needs then to model the interaction of the heavy quarks with the medium, summarized in a few transport coefficients in principle derived from QCD, but for which we are still far from a definite answer for the experimentally relevant conditions. One can then describe the heavy-quark dynamics in the medium: under well-defined kinematic conditions this can be done rigorously through transport equations, which however require the above transport coefficients as an input. The heavy quarks, once they reach a fluid-cell below the deconfinement temperature, undergo hadronization; there is no first-principle theory to model this stage (not even in the vacuum), although one can expect that, at variance with the case of elementary collisions, some sort of recombination with the abundant nearby partons is at work. Clearly this represents an item of interest in itself, but at the same time the related theoretical uncertainties affect the predictions for the final hadronic observables, preventing one from getting an unambiguous information on the partonic stage. Finally, $D$ and $B$ mesons can still rescatter with the surrounding hadrons before reaching kinetic freeze-out: also this possible effect deserves some study. In the following we will discuss the above items in a more quantitative way, eventually considering also to what extent the above setup can be extended to the study of heavy-flavour production in small systems, like high-multiplicity proton-proton/nucleus collisions.

\section{Transport calculations: theoretical setup}
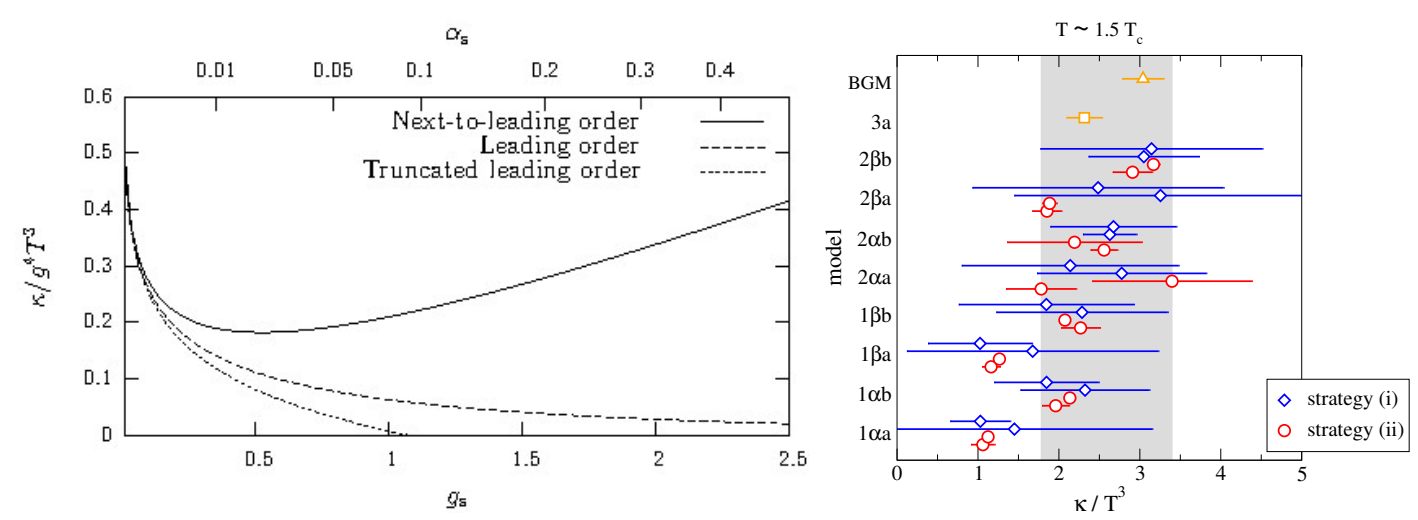

Figure 1: Momentum-diffusion coefficient $k$. Left panel: result of a NLO weak-coupling calculation [1]. Right panel: continuum-extrapolated lattice-QCD results for a gluon plasma [2].

Transport calculations for $c$ and $b$ quarks in heavy-ion collisions are usually formulated in 
terms of the relativistic Langevin equation $[3,4,5]$

$$
\Delta \vec{p} / \Delta t=-\eta_{D}(p) \vec{p}+\vec{\xi}(t) .
$$

In the time-step $\Delta t$ the heavy-quark momentum changes due to a deterministic friction force (quantified by the coefficient $\eta_{D}$ ) and a random noise term $\vec{\xi}$ fixed by its temporal correlator

$$
\left\langle\xi^{i}\left(\vec{p}_{t}\right) \xi^{j}\left(\vec{p}_{t^{\prime}}\right)\right\rangle=b^{i j}\left(\vec{p}_{t}\right) \delta_{t t^{\prime}} / \Delta t, \quad \text { with } \quad b^{i j}(\vec{p}) \equiv \kappa_{\|}(p) \hat{p}^{i} \hat{p}^{j}+\kappa_{\perp}(p)\left(\delta^{i j}-\hat{p}^{i} \hat{p}^{j}\right) .
$$

In the above the transport coefficients $\kappa_{\|}(p)$ and $\kappa_{\perp}(p)$ describe the longitudinal and transverse momentum broadening acquired by the heavy quark while propagating through the medium. In the non-relativistic limit one can ignore the dependence on the heavy-quark momentum and simply set $\kappa_{\|}(p)=\kappa_{\perp}(p) \equiv \kappa$. In such a limit, from the large-time behaviour of the average squared displacement, one can identify the spatial diffusion coefficient $D_{s}$ :

$$
\left\langle\vec{x}^{2}(t)\right\rangle \underset{t \rightarrow \infty}{\sim} 6 D_{s} t \quad \text { with } \quad D_{S}=\frac{2 T^{2}}{\kappa} .
$$

The latter is often used to quantify the strength of the coupling with the medium.

First principle theoretical results are available for $\kappa$ in the static $M \rightarrow \infty$ limit, arising both from analytic weak-coupling calculations [1] and from lattice-QCD simulations [2]. In the left panel of Fig. 1 one can see how, for realistic values of $\alpha_{s}$, the weak-coupling calculation for $\kappa$ receives large NLO corrections, arising mainly from overlapping scattering processes with the light partons from the medium. In the case of lattice-QCD calculations, on the other hand, the right panel of Fig. 1 shows that the final result is affected by large systematic theoretical uncertainties arising from the extraction of real-time information from simulations performed in an Euclidean spacetime. Taking into account the large systematic uncertainties, the two calculations provide results in rough agreement.
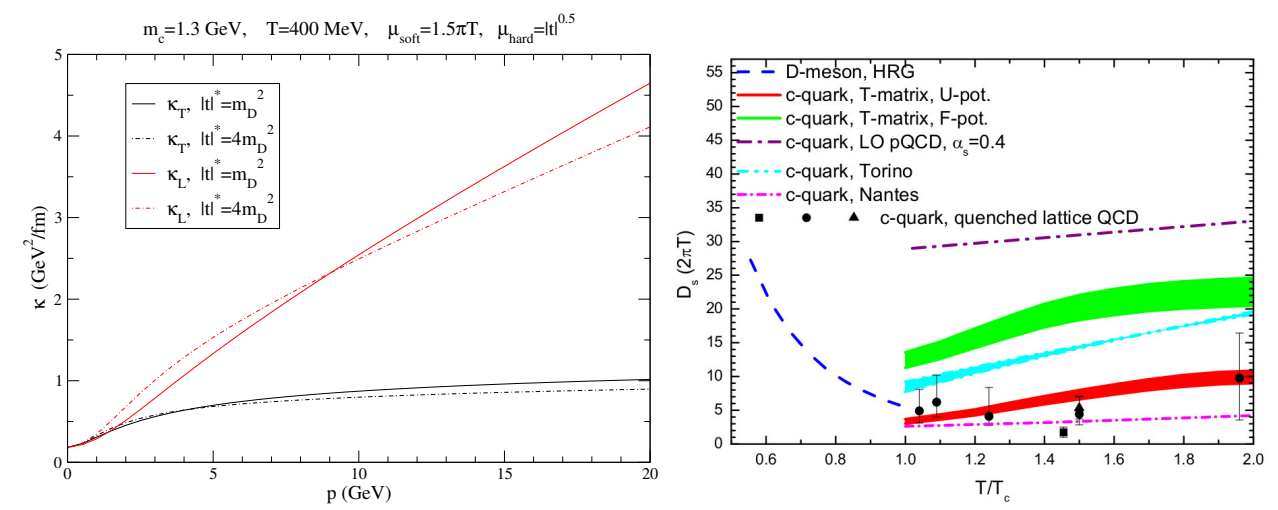

Figure 2: Left panel: charm momentum-broadening coefficients from the weak-coupling calculation in [6]. Right panel: charm spatial diffusion coefficient provided by different theoretical models [7].

Unfortunately, experimentally, the accessible kinematic range for heavy-flavour hadron detection covers mainly relativistic momenta. Hence, transport simulations require a theoretical input going beyond the result of the above static calculations and one needs to consider the full momentum dependence of the coefficients. This was done for instance in [6] with a weak-coupling 
calculation with Hard-Thermal-Loop (HTL) resummation of medium effects: results for $\kappa_{\|}(p)$ and $\kappa_{\perp}(p)$ are displayed in the left panel of Fig. 2. Although the figure clearly shows that the dependence on the particle momentum is relevant and very different for the transverse and the longitudinal coefficients, in comparing the results of different transport calculations one very often simply employs the spatial diffusion coefficient $D_{s}$ defined in Eq. (2.3) to enlighten the differences of the various models; a collection of results for $D_{s}$ obtained under different theoretical frameworks is shown in the right panel of Fig. 2 [7].

\section{Heavy-flavour production in nucleus-nucleus collisions}
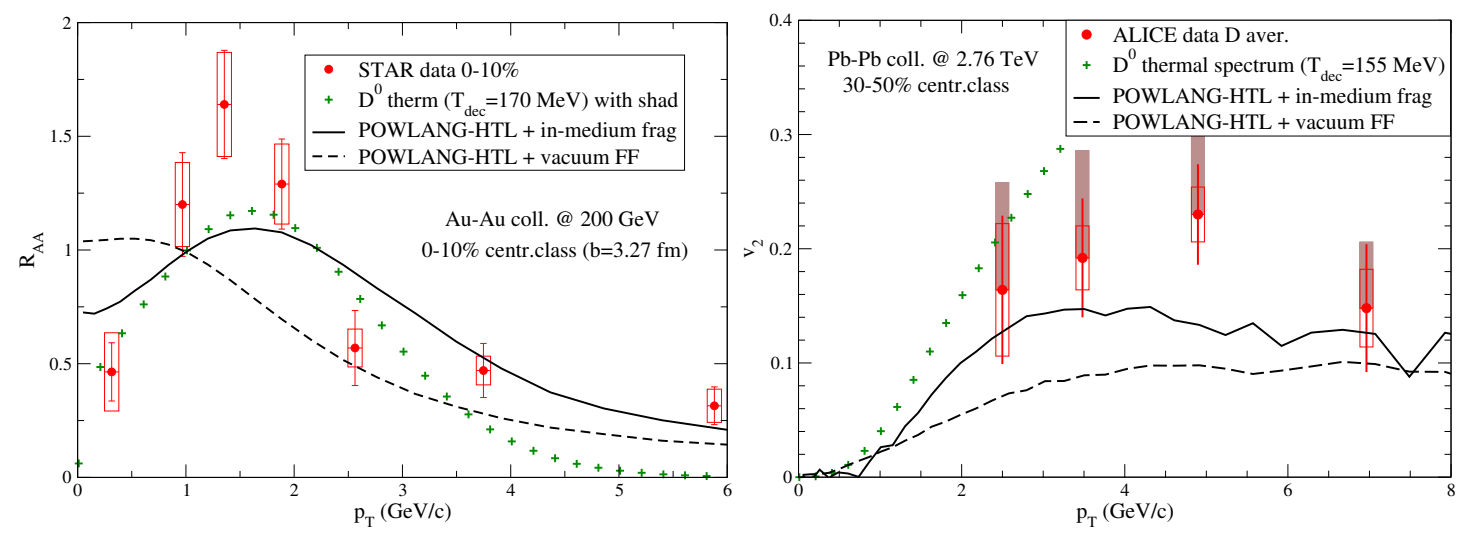

Figure 3: Results for the $D$-meson $R_{\mathrm{AA}}$ and $v_{2}$ in nucleus-nucleus collisions [8] arising from the heavy quark transport (dashed curves) and transport + in-medium hadronization (continuous curves) compared to experimental data $[9,10]$. Green crosses refer to the kinetic-equilibrium limit.

Eq. (2.1), once interfaced with a realistic model for the evolution of the background medium, allows one to study the propagation of heavy quarks throughout the fireball produced in high-energy nuclear collisions. In transport calculations once a heavy quark reaches a fluid cell below the critical deconfinement temperature it is forced to hadronize. While in the vacuum hadronization is usually described in terms of fragmentation functions and leads to an energy degradation of the parent parton, there is evidence that in nuclear collisions recombination with the nearby partons from the medium plays a major role. The process was modeled in several ways in the literature $[11,12,8]$, leading however to similar effects in the particle distributions. In Fig. (3) we display some results of the POWLANG model [8], in which hadronization is modeled via fragmentation of color-singlet string/clusters formed through the recombination of a heavy quark with a light thermal anti-quark from the same fluid-cell. It turns out that the light parton from the medium transfers part of its collective (radial and elliptic) flow to the final $D$-meson, leading to a bump at moderate $p_{T}$ in the $R_{\mathrm{AA}}$ and to an enhancement of the $v_{2}$ and moving theory predictions closer to the experimental data $[9,10]$.

Besides modifying the momentum distribution of the final hadrons, recombination can also change the heavy-flavour hadrochemistry, leading for instance to an enhanced production of $D_{s}$ mesons and $\Lambda_{c}$ baryons. Predictions obtained with models based on the formation of resonant 

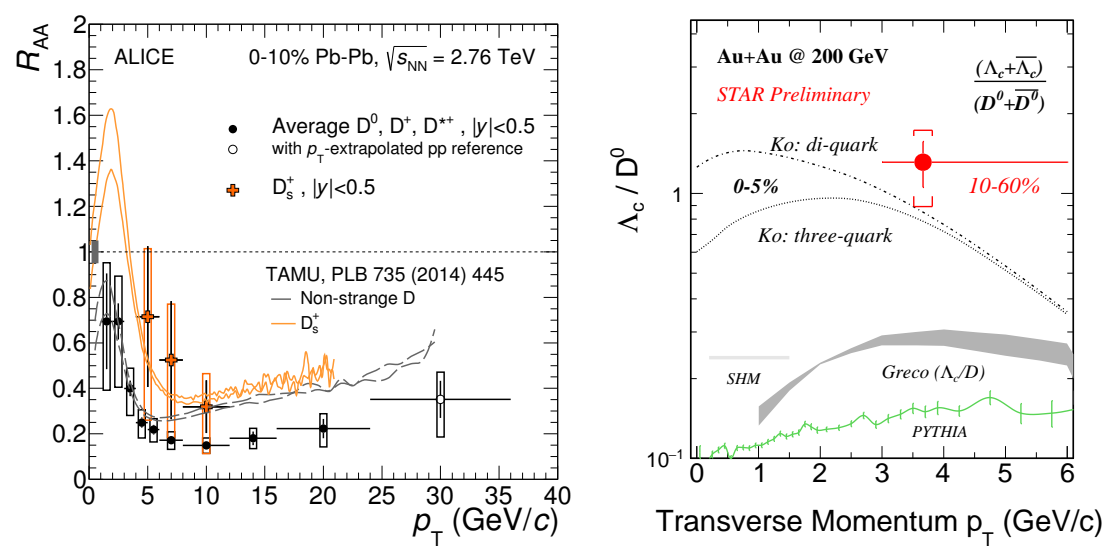

Figure 4: First results for $D_{s}$ [13] and $\Lambda_{c}$ [14] production in nucleus-nucleus collisions compared to transport calculations including in-medium hadronization $[15,16]$.

states around the phase transition [15] and on the coalescence of quarks and di-quarks [16] are shown in Fig. 4 and compared to ALICE [13] and preliminary STAR data [14].
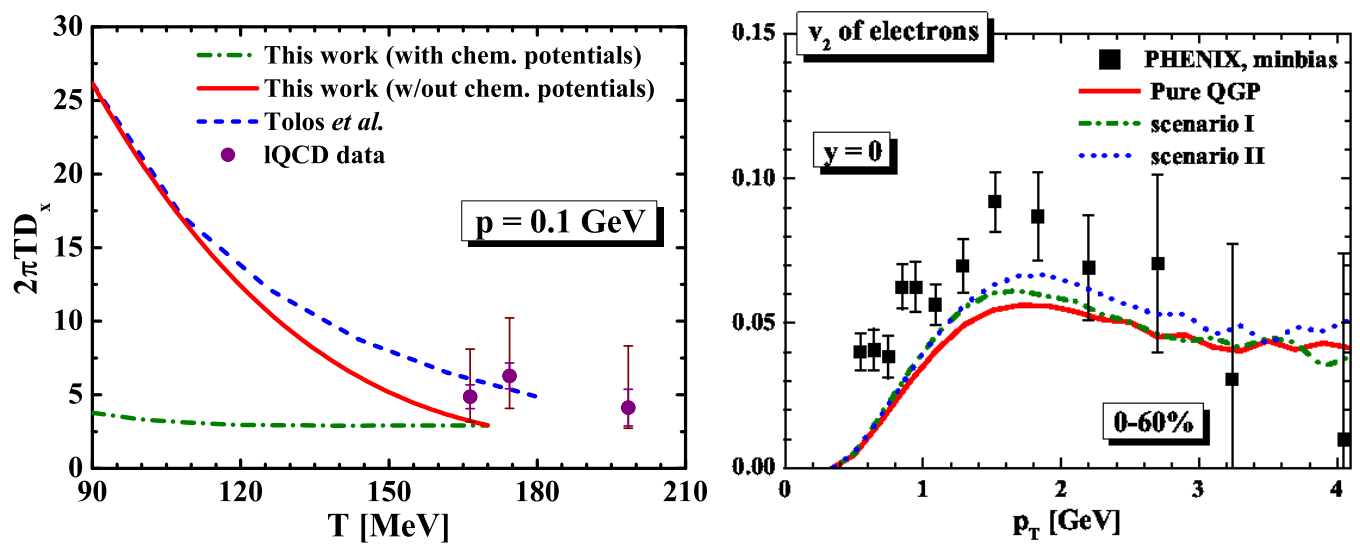

Figure 5: The heavy-quark spatial diffusion coefficient in the hadronic phase (left panel) and its effect on a transport calculation [17] (right panel). Data refer to the heavy-flavour decay electron $v_{2}$ measured in [18].

Finally, although crossing a medium with a lower temperature and hence milder values of the transport coefficients, heavy-flavour particles can suffer rescattering also in the hadronic phase, where the radial and elliptic flow of the fireball is the largest. It is then of interest to evaluate within some effective chiral Lagrangian the transport coefficients of $D / B$-mesons in a gas of light hadrons [17], whose values - as a function of the temperature - turn out to join quite smoothly the results in the partonic phase (left panel of Fig. 5). One can then include also the possibility of rescattering in the hadronic phase in the transport simulations, however the effect on the final observables is found to be quite small, as shown in the right panel of Fig. 5 which refers to the elliptic flow of electrons from semi-leptonic decays of charm and beauty hadrons [18]. 


\section{Heavy-flavour production in small systems}
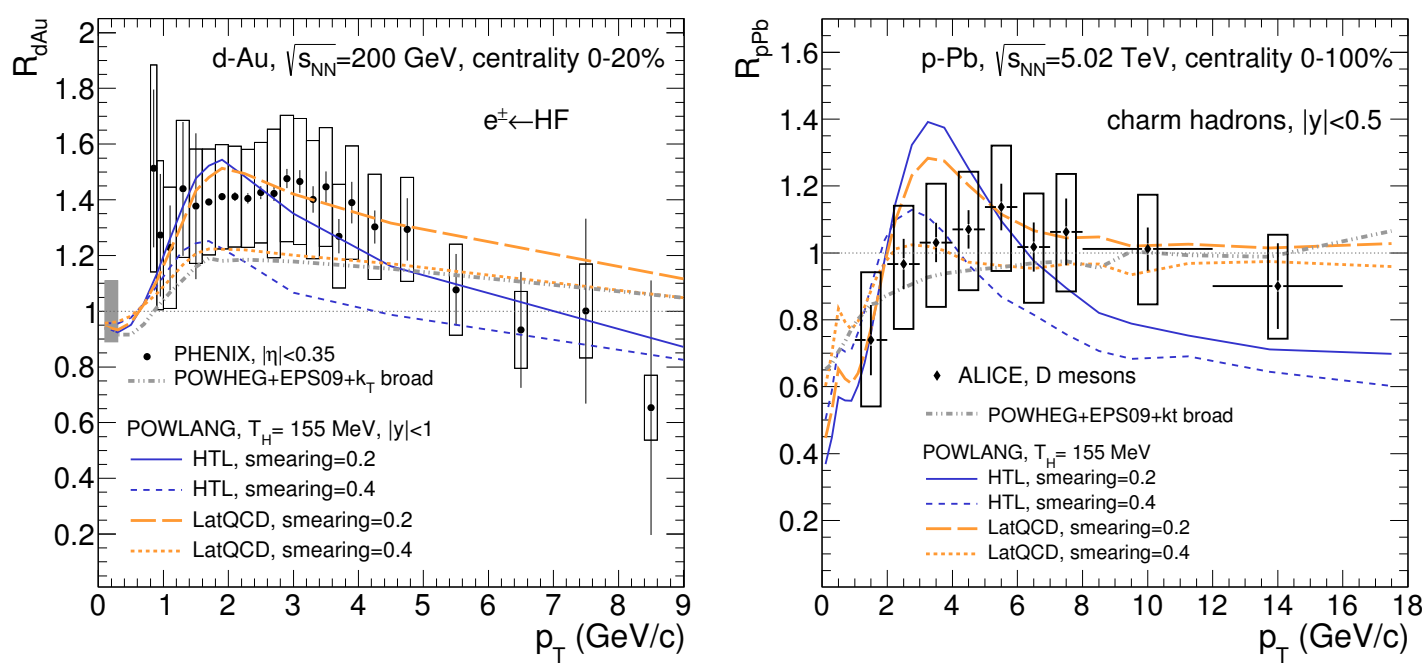

Figure 6: The nuclear modification factor of heavy-flavour decay electrons and $D$-mesons in d-Au and p-Pb collisions at RHIC and LHC. Transport-model results with different transport coefficients [19] are compared to experimental data $[20,21]$.

The observation of signatures of collective effects in measurements of particle correlations performed in high-multiplicity proton-nucleus and proton-proton collisions as well as the universal trend of the integrated particle yields as a function of the final hadron multiplicity led people to wonder about the nature of the possible medium produced in such events. In parallel, no strong evidence of jet-quenching was found in p-p and p-A data: the medium seems to be strongly interacting but not very opaque to highly energetic probes. Hence the interest in studying heavy-flavour production within a transport setup also in the case of small systems. This was done for instance by the POWLANG authors in [19]. Results are shown in Fig. 6, referring to electrons from charm and beauty decays in central d-Au collisions and to charmed hadrons in minimum bias $\mathrm{Pb}-\mathrm{Pb}$ events. Deviations from unity arise from the interplay of several effects: nuclear PDF's, $k_{T}$-broadening in cold nuclear matter, transport in the partonic phase and in-medium hadronization. The large systematic uncertainties of the experimental data $[20,21]$ do not allow one to draw firm conclusions, but leave room for medium effects.

\section{References}

[1] S. Caron-Huot and G. D. Moore, Heavy quark diffusion in QCD and N=4 SYM at next-to-leading order, JHEP 02 (2008) 081, [0801.2173].

[2] A. Francis, O. Kaczmarek, M. Laine, T. Neuhaus and H. Ohno, Nonperturbative estimate of the heavy quark momentum diffusion coefficient, Phys. Rev. D92 (2015) 116003, [1508. 04543 ].

[3] G. D. Moore and D. Teaney, How much do heavy quarks thermalize in a heavy ion collision?, Phys. Rev. C71 (2005) 064904, [hep-ph/ 0412346$].$ 
[4] W. M. Alberico, A. Beraudo, A. De Pace, A. Molinari, M. Monteno, M. Nardi et al., Heavy-flavour spectra in high energy nucleus-nucleus collisions, Eur. Phys. J. C71 (2011) 1666, [1101.6008].

[5] M. He, H. van Hees, P. B. Gossiaux, R. J. Fries and R. Rapp, Relativistic Langevin Dynamics in Expanding Media, Phys. Rev. E88 (2013) 032138, [1305.1425].

[6] W. M. Alberico, A. Beraudo, A. De Pace, A. Molinari, M. Monteno, M. Nardi et al., Heavy flavors in AA collisions: production, transport and final spectra, Eur. Phys. J. C73 (2013) 2481, [1305. 7421$].$

[7] F. Prino and R. Rapp, Open Heavy Flavor in QCD Matter and in Nuclear Collisions, J. Phys. G43 (2016) 093002, [1603.00529].

[8] A. Beraudo, A. De Pace, M. Monteno, M. Nardi and F. Prino, Heavy flavors in heavy-ion collisions: quenching, flow and correlations, Eur. Phys. J. C75 (2015) 121, [1410 . 6082].

[9] STAR collaboration, L. Adamczyk et al., Observation of $D^{0}$ Meson Nuclear Modifications in Au+Au Collisions at $\sqrt{s_{N N}}=200 \hat{a} \breve{A}{ }^{\prime} \hat{A} \breve{A}{ }^{\prime} G e V$, Phys. Rev. Lett. 113 (2014) 142301, [1 404 . 6185].

[10] ALICE collaboration, B. Abelev et al., D meson elliptic flow in non-central Pb-Pb collisions at $\sqrt{s_{\mathrm{NN}}}$ = 2.76TeV, Phys. Rev. Lett. 111 (2013) 102301, [1305.2707].

[11] V. Greco, C. M. Ko and R. Rapp, Quark coalescence for charmed mesons in ultrarelativistic heavy ion collisions, Phys. Lett. B595 (2004) 202-208, [nucl-th/0312100].

[12] P. B. Gossiaux, R. Bierkandt and J. Aichelin, Tomography of a quark gluon plasma at RHIC and LHC energies, Phys. Rev. C79 (2009) 044906, [0901.0946].

[13] ALICE collaboration, J. Adam et al., Measurement of $D_{s}^{+}$production and nuclear modification factor in Pb-Pb collisions at $\sqrt{\mathrm{S}_{\mathrm{NN}}}=2.76 \mathrm{TeV}$, JHEP 03 (2016) 082, [1 509.07287 ].

[14] STAR collaboration, L. Zhou, Measurements of $\Lambda_{c}^{+}$and $D_{s}^{+}$productions in $A u+A u$ collisions at $\sqrt{s_{N N}}=200 \mathrm{GeV}$ from STAR, 1704.04364 .

[15] M. He, R. J. Fries and R. Rapp, Heavy Flavor at the Large Hadron Collider in a Strong Coupling Approach, Phys. Lett. B735 (2014) 445-450, [1401.3817].

[16] Y. Oh, C. M. Ko, S. H. Lee and S. Yasui, Heavy baryon/meson ratios in relativistic heavy ion collisions, Phys. Rev. C79 (2009) 044905, [0901.1382].

[17] V. Ozvenchuk, J. M. Torres-Rincon, P. B. Gossiaux, L. Tolos and J. Aichelin, D-meson propagation in hadronic matter and consequences for heavy-flavor observables in ultrarelativistic heavy-ion collisions, Phys. Rev. C90 (2014) 054909, [1 408 . 4938].

[18] PHENIX collaboration, A. Adare et al., Energy Loss and Flow of Heavy Quarks in Au+Au Collisions at $s(N N) * *(1 / 2)=200-G e V$, Phys. Rev. Lett. 98 (2007) 172301, [nucl-ex/ 0611018 ].

[19] A. Beraudo, A. De Pace, M. Monteno, M. Nardi and F. Prino, Heavy-flavour production in high-energy d-Au and p-Pb collisions, JHEP 03 (2016) 123, [1512.05186].

[20] PHENIX collaboration, A. Adare et al., Cold-nuclear-matter effects on heavy-quark production in $d+$ Au collisions at $\sqrt{s_{N N}}=200 \mathrm{GeV}$, Phys. Rev. Lett. 109 (2012) 242301, [1208 . 1293].

[21] ALICE collaboration, B. B. Abelev et al., Measurement of prompt D-meson production in $p-P b$ collisions at $\sqrt{s_{N N}}=5.02$ TeV, Phys. Rev. Lett. 113 (2014) 232301, [1405. 3452]. 